LARYNGOLOGY

\title{
Efficacy of trans-nasal fiberendoscopic injection laryngoplasty with centrifuged autologous fat in the treatment of glottic insufficiency due to unilateral vocal fold paralysis
}

\author{
Efficacia della laringoplastica iniettiva fibroendoscopica trans-nasale con grasso \\ autologo centrifugato nel trattamento dell'insufficienza glottica da paralisi cordale \\ monolaterale
}

\author{
A. RICCI MACCARINI ${ }^{1}$, M. STACCHINI ${ }^{1}$, F. MOZZANICA ${ }^{2}$, A. SCHINDLER ${ }^{2}$, E. BASILE ${ }^{3}$, G. DE ROSSI ${ }^{4}$, P. WOO ${ }^{5}$, \\ M. REMACLE ${ }^{6}$, M. MAGNANI ${ }^{1}$ \\ ${ }^{1}$ ENT Department, M. Bufalini Hospital, Cesena, Italy; ${ }^{2}$ Department of Biomedical and Clinical Sciences, L. Sacco \\ Hospital, University of Milan, Milan, Italy; ${ }^{3}$ ENT Clinic, University of Messina, Messina, Italy; ${ }^{4}$ Medical Center \\ of Phoniatrics and Phonosurgery, Padua, Italy; ${ }^{5}$ Clinical Professor, Department Of Otolaryngology Head and Neck \\ Surgery, Icahn School of Medicine, New York, USA; ${ }^{6}$ Department of Otorhinolaryngology Head and Neck Surgery, Centre \\ Hospitalier Luxemburg, Luxemburg
}

\begin{abstract}
SUMMARY
The objective of this work is to evaluate the safety, feasibility and efficacy of trans-nasal fiberendoscopic injection laryngoplasty (IL) with centrifuged autologous fat, performed under local anaesthesia, in the treatment of glottic insufficiency due to unilateral vocal fold paralysis (UVFP). It is a within-subject study with follow-up 1 week after phonosurgery and after 6 months. A total of 22 patients with chronic dysphonia caused by glottic insufficiency due to UVFP were enrolled. Each patient underwent trans-nasal IL with centrifuged autologous fat through flexible operative endoscope under local anaesthesia and was evaluated before and twice (1 week and 6 months) after phonosurgery, using a multidimensional set of investigations. The assessment protocol included videolaryngostroboscopy, perceptual evaluation of dysphonia, maximum phonation time and patient's self-assessment on voice-related quality of life (QOL) with the Voice Handicap Index-10 and the comparative self-assessment on vocal fatigue and voice quality pre-post treatment. Trans-nasal IL with centrifuged autologous fat was performed in all 22 patients and there were no complications in any case. Significant improvements in videolaryngostroboscopic findings, perceptual evaluation of dysphonia, maximum phonation time and QoL self-assessment were reported after 1 week and were maintained at 6 months. In one patient, the result after 6 months was not satisfactory and this patient then underwent a medialization laryngoplasty (thyroplasty type I) with satisfactory long-term results. In conclusion, trans-nasal fiberendoscopic IL with centrifuged autologous fat seems to be a safe, feasible and efficacious phonosurgical procedure for treatment of glottic insufficiency due to unilateral vocal fold paralysis.
\end{abstract}

KEY WORDS: Injection laryngoplasty • Autologous fat injection • Phonosurgery under local anaesthesia • Trans-nasal fiberendoscopic phonosurgery $\bullet$ Vocal fold paralysis

\begin{abstract}
RIASSUNTO
L'obiettivo di questo lavoro è quello di valutare la sicurezza, la fattibilità e l'efficacia della laringoplastica iniettiva (LI) fibroendoscopica trans-nasale con grasso autologo centrifugato, effettuata in anestesia locale, nel trattamento della insufficienza glottica da paralisi cordale monolaterale (PCML). È uno studio intra-individuale con controlli a una settimana e 6 mesi dopo la fonochirurgia. Riguardo ai metodi, sono stati arruolati nello studio 22 pazienti con disfonia cronica causata da insufficienza glottica da PCML. Ogni paziente è stato sottoposto a LI trans-nasale con grasso autologo centrifugato mediante endoscopio flessibile operativo in anestesia locale ed è stato valutato prima e a distanza di una settimana e 6 mesi dall'intervento di fonochirurgia, utilizzando una serie di indagini multidimensionale. Il protocollo valutativo comprendeva la videolaringostroboscopia, la valutazione percettiva della disfonia, il tempo massimo fonatorio e l'autovalutazione da parte del paziente sulla qualità della vita in relazione al problema vocale mediante il Voice Handicap Index-10 e l'Autovalutazione Comparativa pre-post trattamento riguardo alla fatica fonatoria e alla qualità della voce. I risultati hanno mostrato che la LI trans-nasale con grasso autologo centrifugato effettuata nei 22 pazienti non ha avuto complicanze. Sono stati riportati miglioramenti significativi dei rilievi laringostroboscopici, della valutazione percettiva della disfonia, del tempo massimo fonatorio e dell'autovalutazione sulla qualità della vita ad una settimana dall'intervento di fonochirurgia, che si sono mantenuti anche a distanza di 6 mesi. In un paziente il risultato dopo 6 mesi non è stato soddisfacente ed è stato operato di laringoplastica di medializzazione cordale (tiroplastica di tipo I) con soddisfacenti risultati a lungo termine. In conclusione, la LI fibroendoscopica trans-nasale con grasso autologo centrifugato sembra essere una procedura fonochirurgica sicura, fattibile ed efficace per il trattamento dell'insufficienza glottica da paralisi cordale monolaterale.
\end{abstract}

PAROLE CHIAVE: Laringoplastica iniettiva • Iniezione di grasso autologo • Fonochirurgia in anestesia locale • Fonochirurgia fibroendoscopica trans-nasale $\bullet$ Paralisi della corda vocale

Acta Otorhinolaryngol Ital 2018;38:204-213 


\section{Introduction}

Unilateral vocal fold paralysis (UVFP) is a frequent complication of surgical manipulation of the thyroid gland and mediastinal structures, which are located along the pathway of the recurrent laryngeal nerve ${ }^{1-3}$. Less than $50 \%$ of cases of vocal fold immobility caused by injury during surgery recover spontaneously within one year, while the remaining patients show persistent UVFP ${ }^{1}$. A growing number of idiopathic UVFP is documented ${ }^{2-4}$. In case of UVFP glottic insufficiency (GI) often occurs, which causes dysphonia and dysphagia and reduces patient's quality of life ${ }^{5}$. In addition, GI due to UVFP may significantly compromise postoperative recovery in patients with decreased pulmonary reserve or inability to protect their airways ${ }^{6}$.

Voice therapy is usually the first choice in the treatment of GI due to UVFP. Its aim is to improve glottic compensation by the contralateral mobile vocal fold ${ }^{7}$.

When voice therapy does not provide a positive effect, in particular when the glottic gap is wide because the immobile vocal fold (VF) lies in abducted (lateral) or intermediate position, phonosurgery may be indicated ${ }^{8}$. Several phonosurgical techniques for the treatment of GI due to UVFP have been proposed, which include medialisation laryngoplasty (thyroplasty type I) and/or arythenoid adduction ${ }^{9}$ and neurorrhaphy ${ }^{10}$. Injection laryngoplasty (IL) is another widely applied phonosurgical technique ${ }^{8}$. IL is a safe and well-established procedure, frequently performed in the early treatment of GI ${ }^{810}$. Previous studies have shown that the improvement of glottic valvular function obtained with IL could improve symptoms of dysphonia and dysphagia ${ }^{611-14}$. In addition, early medialisation IL within 6 months of onset of UVFP may reduce the need of permanent external phonosurgery ${ }^{614}$.

IL can be performed either under local or general anaesthesia using various alloplastic materials ${ }^{10}{ }^{14}$ or autologous fat ${ }^{8}$. The advantages of the local anaesthesia (LA) are noteworthy. First of all, evaluating and treating patients when they are awake and able to phonate allows a "functional" visualisation of the operative field, allows the phonosurgeon to monitor the effects of IL during and after the intervention. Moreover, IL under LA reduces costs of the procedure compared to a direct microlaryngoscopy procedure ${ }^{15-17}$. Finally, IL under LA may also be performed in patients with difficult laryngeal exposure and in patients with reduced neck extension or abnormal neck anatomy ${ }^{811}$. Several routes to reach the vocal fold (VF) during IL have been described: through the cricothyroid membrane or through the thyrohyoid membranes, or through a trans-oral approach ${ }^{10}$. These procedures may be difficult to perform, require great patient tolerance and may be impossible to perform when anatomic or physiologic barriers exist ${ }^{15}$. In order to overcome these limitations, a phonosurgical approach using a trans-nasal flexible fiberendoscope has been proposed by the CELF group (Cirugia EndoLaringea Fibroscopica) of Santander (Spain) ${ }^{18}$ and further developed in Italy ${ }^{81920}$. This phonosurgical technique is also used for the removal of laryngeal polyps, granulomas and papillomas with flexible cold instruments or with fibre lasers ${ }^{20-22}$ as well as to perform IL ${ }^{8}$; it was named "fiberendoscopic phonosurgery" (FEPS) ${ }^{81920}$. In particular, regarding IL under FEPS, Ricci-Maccarini et al. ${ }^{819}$ developed a completely transnasal fiberendoscopic IL procedure, using a high-pressure injection pistol connected to a flexible injection needle, injecting centrifuged autologous fat (with modified Coleman technique) 58192324 . This procedure can also be employed for the IL with other materials and in particular hyaluronic acid ${ }^{8}$. The use of autologous fat reduces the risk of allergic reactions and local granulomatosis that could be caused by synthetic materials ${ }^{811}$. The centrifuged autologous fat as an IL material gives the advantage of less reabsorption compared to other injectable materials like hyaluronic acid, because the stem cells contained in the adipose stroma regenerate new lipocytes which replace the lipocytes deteriorated by the injection procedure ${ }^{23-25}$.

Preliminary results on the efficacy of trans-nasal IL in the treatment of glottic insufficiency due to UVFP ${ }^{11} 15$ seem promising, although only scarce information regarding its safety, feasibility and efficacy in the treatment of secondary dysphonia caused by UVFP both in the short- and in the long-term period are available.

The aim of this study is to evaluate the safety, feasibility and efficacy of trans-nasal IL with centrifuged autologous fat under fiberendoscopy in the treatment of glottic insufficiency due to UVFP. The underling hypothesis is that this phonosurgical technique is simple, safe, reproducible and could provide satisfactory results, in both the shortand in the long-term periods. The importance of this study is related to the need for a simple and effective procedure to treat GI due to UVFP which does not recover with voice therapy.

\section{Materials and methods}

\section{Participants}

A total of 22 patients, 11 females and 11 males, with at least 6 months history of dysphonia caused by GI due to UVFP were enrolled. The mean age was $60 \pm 13$ years 
(range 32-79). The aetiology of UVFP is reported in Table I. Each patient underwent voice therapy for at least 2 months after the onset of dysphonia caused by GI due to UVFP, with the aim of improving glottic compensation by the contralateral mobile vocal fold (VF). When voice therapy was unable to obtain a satisfactory result, the patient underwent trans-nasal fiberendoscopic IL with centrifuged autologous fat (CAF). Each procedure was performed in the operating room (OR) after formal written consent was obtained. In our institution, IL under fiberendoscopy is usually performed in an office-based setting using hyaluronic acid, but when autologous fat is injected, an OR setting is preferred as centrifuged autologous fat needs to be manipulated in a sterile environment; an anaesthesiologist is not required, and the patient is monitored to control for eventual vagal nerve reflex problems.

\section{Surgical procedure}

IL was performed under LA on a day hospital basis. All procedures were performed by two surgeons: one manoeuvered the fiberscope and the other one manoeuvered the flexible injection needle and high-pressure pistol.

Table I. Demographic characteristics of the patients, aetiology of unilateral vocal fold paralysis.

\begin{tabular}{|c|c|c|c|c|}
\hline Patient N. & Sex & Age (years) & Aetiology of UVFP & Side of injection \\
\hline 1 & $\mathrm{~F}$ & 65 & T & Left \\
\hline 2 & $F$ & 77 & I & Right \\
\hline 3 & M & 65 & $P$ & Left \\
\hline 4 & $F$ & 38 & I & Right \\
\hline 5 & $F$ & 73 & T & Right \\
\hline 6 & $F$ & 32 & T & Right \\
\hline 7 & M & 57 & $P$ & Left \\
\hline 8 & M & 53 & $P$ & Left \\
\hline 9 & $F$ & 54 & T & Left \\
\hline 10 & $\mathrm{~F}$ & 47 & T & Left \\
\hline 11 & M & 59 & $P$ & Left \\
\hline 12 & $F$ & 38 & I & Left \\
\hline 13 & M & 78 & T & Left \\
\hline 14 & M & 69 & T & Right \\
\hline 15 & M & 64 & T & Right \\
\hline 16 & M & 64 & T & Right \\
\hline 17 & M & 70 & $P$ & Left \\
\hline 18 & M & 51 & T & Left \\
\hline 19 & M & 58 & T & Right \\
\hline 20 & $F$ & 71 & T & Right \\
\hline 21 & $F$ & 79 & I & Left \\
\hline 22 & $\mathrm{~F}$ & 49 & $T$ & Left \\
\hline
\end{tabular}

T: post-thyroidectomy; P: post-pneumonectomy; l: idiopathic) and side of injection.
Thirty minutes before the surgical procedure each patient received intramuscular atropine $(0.5 \mathrm{mg})$ to reduce salivary secretions on the vocal folds and vagal nerve reflexes (bradycardia, lipothymia). The patient was initially placed in a supine position. An infiltration of local anaesthetic and vasoconstrictor solution was administered into the low peri-umbilical region, using two $20 \mathrm{cc}$ syringes connected to a 22 gauge needle, $9 \mathrm{~cm}$ long. After 20 minutes, intravenous injection of $1 \mathrm{mg}$ of midazolam was administered and liposuction was performed in the sub-cutis of the low peri-umbilical region, using a $10 \mathrm{cc}$ disposable autostatic syringe, connected via luer-lock to a 14 gauge Chiba needle, $9 \mathrm{~cm}$ long (instead of the Coleman cannula). Lipoaspirate concentration was achieved by centrifugation for 3 minutes at $3000 \mathrm{rpm}$ as described by Coleman ${ }^{23}$. This technique also concentrates the stromal stem cells contained in the adipose tissue, which may favour the regeneration of the infiltrated fat tissue, maintaining its volume ${ }^{24}$. The serum was eliminated from the centrifuged material and the concentrated fat (without mixing it with other substances) was placed into a disposable $3 \mathrm{cc}$ luer-lock polycarbonate syringe, which was then inserted into a high-pressure injection pistol (modified Uroplasty pistol) ${ }^{8}$. Once this task was completed, the patient was placed in a semi-seated position, with his/her head tilted slightly backwards, to facilitate the sight of the glottic plane. Local anaesthesia was administered with vaporisation of $10 \%$ lidocaine into the nose and pharynx. The operative flexible endoscope was inserted trans-nasally; lidocaine $2 \%$ and then $10 \%$ was instilled into the larynx through the working channel of the fiberscope, using a plastic catheter (BTC Medical Europe). In difficult patients also a trans-cutaneous infiltration of lidocaine into the site of the superior laryngeal nerve, as in a thyroplasty procedure ${ }^{9}$ can be helpful.

A flexible endoscope with a $2 \mathrm{~mm}$ working channel, an outer diameter of $5 \mathrm{~mm}$ and working length of $23 \mathrm{~cm}$ (Storz 11001UD1, Tuttlingen, Germany) was used in all surgical procedures. For fat injection, a 19 gauge endoscopic flexible needle, $80 \mathrm{~cm}$ in length (BTC Medical Europe, Valeggio sul Mincio, Verona, Italy) was used. The endoscopic needle was linked to the high-pressure injection pistol manoeuvered by the second operator. A single injection was performed laterally to the vocal process of the immobile vocal fold to obtain a vocal fold medialisation and augmentation (Figs. 1, 2). Because up to $50 \%$ of the injected fat might be reabsorbed within some months, double amount of fat than the amount needed to correct glottic insufficiency was injected ${ }^{819}$. In none of the cases was a bilateral VF injection performed. 


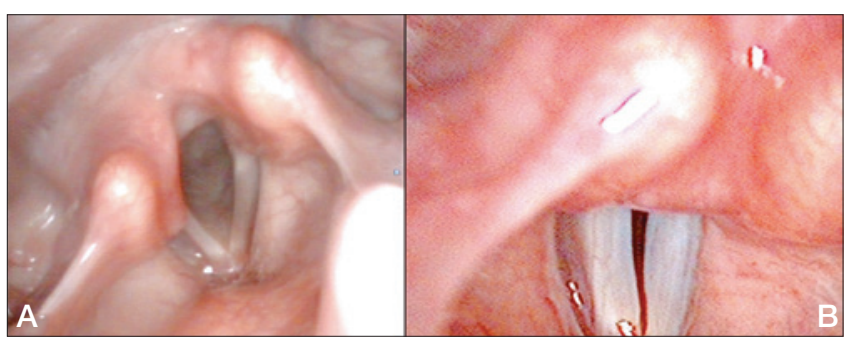

Fig. 1. Clinical case, Female, 32 years old. pre-op. GRBS scale: G2, R1, B2, A2, So. Laryngostroboscopy: a) VF during breathing, the right VF is atrophic and immobile in abducted position; b) VF during phonation, glottic closure is incomplete with large gap, periodicity of glottic vibration is irregular; MPT: 6 sec.; VHI-10: 21.

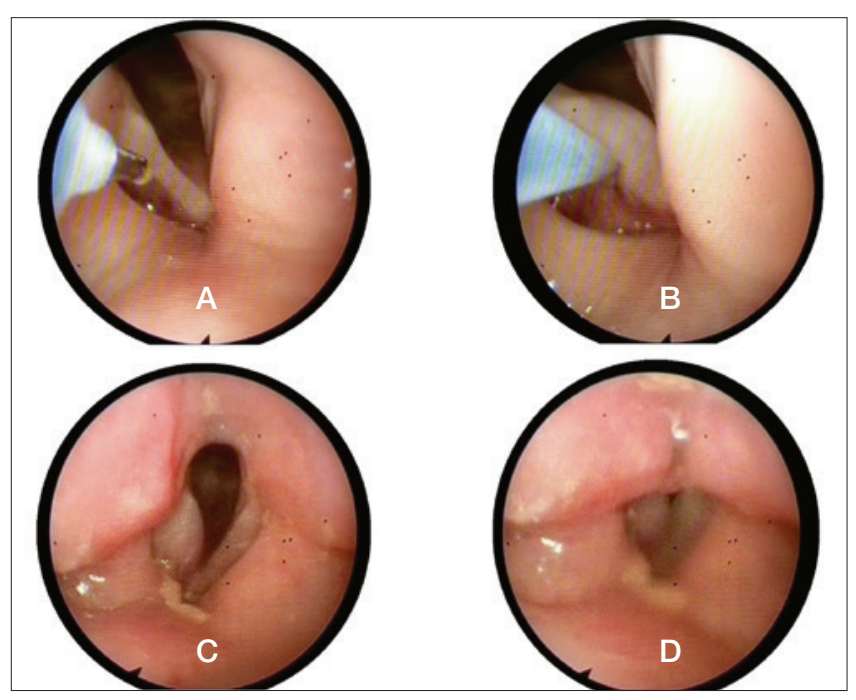

Fig. 2. Same clinical case of Fig. 1, Phonosurgery: injection laryngoplasty with centrifuged autologous fat; a) approximation of the fiberscope to the immobile right VF with the 19 Gauge flexible needle outside the working channel; b) injection of centrifuged autologous fat into the immobile right VF; c) VF during breathing at the end of intervention; d) VF during phonation at the end of intervention, glottic closure is almost complete with a small posterior gap due to the convex profile of the injected VF.

\section{Patient evaluation}

The number and type of complications during and after phonosurgery were analysed. Each patient was evaluated before, after 1 week (first post-operative-period) (Fig. 3) and after 6 months (second post-operative-period) (Fig. 4) from phonosurgery using a multidimensional set of measurements according to the guidelines of the European Laryngological Society ${ }^{26}$. In particular, each patient underwent videolaryngostroboscopy with either a rigid or flexible endoscope. The exam was recorded on video, stored in the host computer and then scored jointly by two phoniatricians blind to timing of the sur-

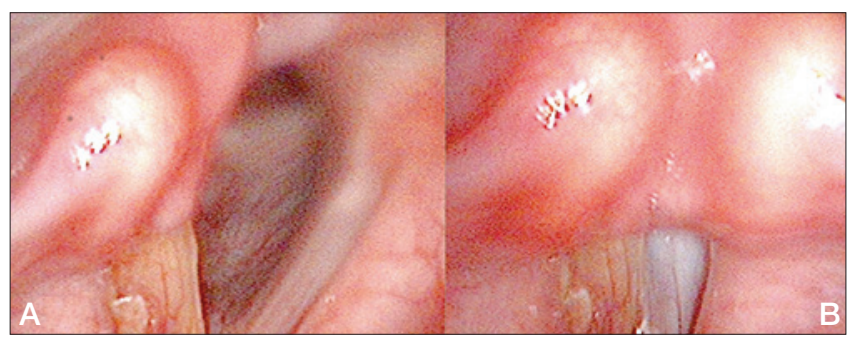

Fig. 3. Same clinical case of Figs. 1 and 2; one week post-op. GRBS scale: GO. Laryngostroboscopy: a) VF during breathing, the right VF is hypertrophic and immobile in a paramedian position; b) VF during phonation, glottic closure is complete, periodicity of glottic vibration is regular; MPT: $14 \mathrm{sec}$.; VHI-10: 8; comparative self-assessment: great improvement both for voice quality and vocal fatigue.

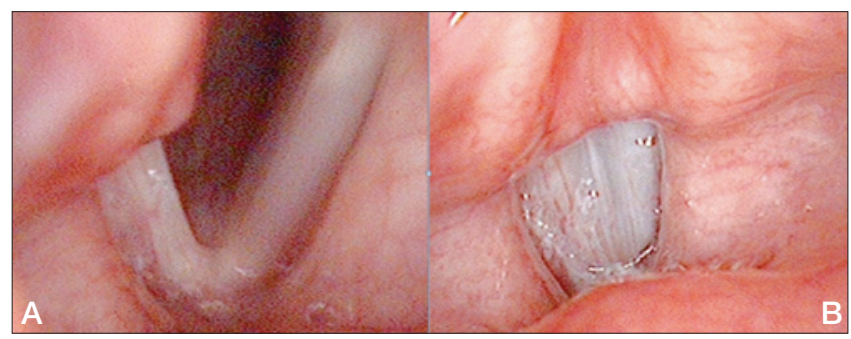

Fig. 4. Same clinical case of Figs. 1, 2 and 3; six months post-op. GRBAS scale: G0. Laryngostroboscopy: a) VF during breathing, the right VF has a normal morphology and it is immobile in an intermediate position; b) VF during phonation, glottic closure is complete although the immobile right VF is less medialised than in the first post-op. control, periodicity of glottic vibration is regular; MPT: 12 sec.; VHI-10: 5 ; comparative self-assessment: great improvement both for voice quality and vocal fatigue.

gical procedure, who finally discussed the results of the examination in order to assign an univocal score. During videolaryngostroboscopic examination, glottic closure (which was scored as: complete, incomplete with small gap, incomplete with large gap), the position of the immobile vocal fold (which was scored as: immobile in median, paramedian, intermediate, abducted position or it could be normally mobile), the morphology of the vocal folds (which was scored as: normal, atrophic, hypertrophic) and the periodicity of glottic vibration (which was scored as: regular, irregular, inconsistent) were assessed, according to Hirano's nomenclature ${ }^{27}$ and its modified updated version ${ }^{28}$. For the perceptual evaluation of dysphonia the GRBAS scale was used ${ }^{27-29}$. The conversational speech on standard sentences of each patient were recorded on the host computer. The recordings were rated jointly by an experienced phoniatrician and an experienced speech pathologist, both blind to the timing of the phonosurgical procedure and assessment, who discussed the results of the analysis in order to assign a univocal score to each of 
the items included in the GRBAS scale. For the recording of voice signal, a microphone approximately $15 \mathrm{~cm}$ from the voice source was used to avoid an airflow effect. For aerodynamic evaluation, the maximum phonation time (MPT) was measured: each patient was asked to utter an/a/ in modal register, as long as he/she could. MPT was determined by measuring the sustained/a/ in 3 productions on the basis of the oscillogram signal (abnormal values $<10$ seconds ${ }^{27}$ ). To obtain self-assessment data on perceived QOL, each patient completed the Italian version of the VHI- $10^{30}$ (abnormal values $>11^{31}$ ). Moreover, to obtain information from the patient regarding his/her perceived modification of voice after phonosurgery, each patient was asked to rate the "comparative self-assessment pre-post-treatment" regarding the changes of voice quality and vocal fatigue by means of a 5 points scale $(-2=$ great worsening; $-1=$ slight worsening; $0=$ no difference $;+1=$ slight improvement $;+2=$ great improvement) ${ }^{28}$.

\section{Statistical analysis}

The results are presented as arithmetic mean \pm standard deviation. The Kruskal-Wallis test with Mann-Whitney post hoc for continuous variables and Fisher test for categorical variables were used to test the mean difference among the pre- and the post-operative periods. A significance level of 0.05 for all testing was used. Statistical analyses were performed using the SPSS 21.0 package (SPSS Science, Chicago, IL, USA).

\section{Ethical approval}

All procedures performed in studies involving human participants were in accordance with the ethical standards of the institutional and/or national research committee and with the 1964 Helsinki declaration and its later amendments or comparable ethical standards.

\section{Results}

Trans-nasal fiberendoscopic IL with CAF was performed in all 22 patients. The liposuction and the phonosurgical procedure were well tolerated by all patients. In none of the cases was any complication during the procedure or difficulties in the trans-nasal passage of the fiberscope reported. In particular, no haemorrhage in the nose or at the injection site was noted intra- or post-operatively; no granuloma was visible at the injection site after 1 week and 6 months. The amount of the injected fat was ranged between $2 \mathrm{cc}$ to $3 \mathrm{cc}$ for each injected VF; IL was always unilateral in all the treated patients. As far as categorical variables are concerned, Fisher's test demonstrated a significant difference in the videolaryngostroboscopic findings in the pre- and the post-operative periods for all variables (Table II). In particular, in the pre-operative period the morphology of the immobile vocal fold was considered normal in 1 of the 9 immobile right VF and in none of the 13 immobile left VF, while VF atrophy was found in the remaining 21 immobile VF of the 22 patients. In the first post-operative period 7 of the 9 immobile right VF and 9 of the 13 immobile left VF had a normal morphology; VF hypertrophy was found in 2 of the 9 immobile right VF and in 4 of the 13 immobile left VF, while in none of the cases vocal fold atrophy was detected. This difference was significant by Fisher's test ( $p=0.001$ and $\mathrm{p}=0.000$ for the right and left morphology, respectively). In the second post-operative period, 8 of the 9 immobile right VF and 12 of the 13 immobile left VF were considered normal, while VF atrophy was detected in only 1 right and 1 left immobile VF. No significant difference of vocal fold morphology between the first and the second post-operative periods was demonstrated on Fisher test. The position of the immobile VF and the periodicity of glottic vibration significantly improved after phonosurgery and no significant differences between the first and the second post-operative periods were found on Fisher's analysis.

Glottic closure also significantly improved after phonosurgery. In the first post-operative period complete glottic closure was achieved in 20 patients while an incomplete glottic closure with small gap was found in 2 patients, where a large gap was reported in the pre-operative period. In the second post-operative period, complete glottic closure was achieved in 15 patients, while in the remaining 7 patients incomplete glottic closure with a small gap was found. These differences were significant by Fisher's test $(\mathrm{p}=0.047)$.

As far as continuous variables are concerned, the KruskalWallis test demonstrated a significant difference in the scores obtained among the pre- and the post-operative periods for all variables. In particular, the perceptual evaluation of dysphonia demonstrated significant improvement in both the first and in the second post-operative periods for all the GRBA parameters (Table III). The S parameter was never altered even in the pre-operative period ( $p=0.317)$ because all patients had an asthenic voice before phonosurgery and never a strained voice. No differences in the GRBAS scale were seen between the first and the second post-operative periods, with the exception of the $\mathrm{R}$ parameter, which was worst at one week after phonosurgery and was further improved in the second postoperative period (Table III).

The MPT score was significantly increased both in the 
Table II. Videolaryngostroboscopic examination of patients: VF morphology, position of the immobile VF, periodicity of glottic vibration and glottic closure are reported. The results of Fisher's test as well as those of the post-hoc analysis are reported.

\begin{tabular}{|c|c|c|c|c|c|c|c|}
\hline & & Pre & $1^{\text {st }}$ post & $2^{\text {nd }}$ post & & Fisher tes & \\
\hline & & & & & $\begin{array}{l}\text { Pre vs } \\
1^{\text {st }} \text { post }\end{array}$ & $\begin{array}{c}\text { Pre vs } \\
2^{\text {nd }} \text { post }\end{array}$ & $\begin{array}{c}1^{\text {st }} v s \\
2^{\text {nd }} \text { post }\end{array}$ \\
\hline $\begin{array}{l}\text { Morphology } \\
\text { Right VF }\end{array}$ & Normal & $\begin{array}{l}14 \\
(1)\end{array}$ & $\begin{array}{l}20 \\
(7)\end{array}$ & $\begin{array}{l}21 \\
(8)\end{array}$ & 0.001 & 0.002 & 0.230 \\
\hline & Atrophic & $\begin{array}{c}8 \\
(8)\end{array}$ & 0 & $\begin{array}{c}1 \\
(1)\end{array}$ & & & \\
\hline & Hypertrophic & 0 & $\begin{array}{c}2 \\
(2)\end{array}$ & 0 & & & \\
\hline $\begin{array}{l}\text { Morphology } \\
\text { Left VF }\end{array}$ & Normal & $\begin{array}{c}9 \\
(0)\end{array}$ & $\begin{array}{l}18 \\
(9)\end{array}$ & $\begin{array}{l}21 \\
(12)\end{array}$ & 0.001 & 0.001 & 0.084 \\
\hline & Atrophic & $\begin{array}{c}13 \\
(13)\end{array}$ & 0 & $\begin{array}{l}1 \\
(1)\end{array}$ & & & \\
\hline & Hypertrophic & 0 & $\begin{array}{c}4 \\
(4)\end{array}$ & 0 & & & \\
\hline Position & Mobile & 13 & 13 & 13 & 0.006 & 0.010 & 0.399 \\
\hline & $\begin{array}{l}\text { Immobile } \\
\text { Median }\end{array}$ & 0 & 2 & 0 & & & \\
\hline & $\begin{array}{c}\text { Immobile } \\
\text { Paramedian }\end{array}$ & 1 & 7 & 8 & & & \\
\hline & $\begin{array}{l}\text { Immobile } \\
\text { Intermediate }\end{array}$ & 4 & 0 & 1 & & & \\
\hline & $\begin{array}{l}\text { Immobile } \\
\text { Abducted }\end{array}$ & 4 & 0 & 0 & & & \\
\hline Position & Mobile & 9 & 9 & 9 & 0.001 & 0.003 & 0.690 \\
\hline & $\begin{array}{l}\text { Immobile } \\
\text { Median }\end{array}$ & 0 & 5 & 3 & & & \\
\hline & $\begin{array}{l}\text { Immobile } \\
\text { Paramedian }\end{array}$ & 2 & 8 & 9 & & & \\
\hline & $\begin{array}{l}\text { Immobile } \\
\text { Intermediate }\end{array}$ & 6 & 0 & 1 & & & \\
\hline & $\begin{array}{l}\text { Immobile } \\
\text { Abducted }\end{array}$ & 5 & 0 & 0 & & & \\
\hline Periodicity of glottic & Normal & 3 & 11 & 16 & 0.001 & 0.001 & 0.158 \\
\hline & Irregular & 14 & 11 & 6 & & & \\
\hline & Inconsistent & 5 & 0 & 0 & & & \\
\hline Glottic closure & Complete & 0 & 20 & 15 & 0.001 & 0.001 & 0.047 \\
\hline & $\begin{array}{l}\text { Incomplete } \\
\text { small gap }\end{array}$ & 6 & 2 & 7 & & & \\
\hline & $\begin{array}{l}\text { Incomplete } \\
\text { large gap }\end{array}$ & 16 & 0 & 0 & & & \\
\hline
\end{tabular}

Pre: pre-operative period; 1 ${ }^{\text {st }}$ post: post-operative period 1 week; $2^{\text {nd }}$ post: post-operative period 6 months (bracketing the number of the immobile VF).

first and in the second post-operative periods ( $\mathrm{p}=0.001$ at Kruskal-Wallis test). In particular, the MPT was scored $5.63 \pm 4.44$ in the pre-operative period, while it was $8.82 \pm 3.25$ and $10.55 \pm 4.97$ in the first and in the second post-operative periods, respectively. Post-hoc analysis demonstrated significant differences between the pre-operative and the first post-operative periods $(\mathrm{p}=0.001)$ and between the pre-operative and the second post-operative periods $(\mathrm{p}=0.001)$. No differences between the MPT results obtained in the first and second post-operative peri- 
Table III. Results of perceptual evaluation of dysphonia in the pre- and the 2 post-treatment periods. The results of the Kruskal-Wallis test as well as those of the post-hoc analysis (performed using Mann-Whitney test) are also reported.

\begin{tabular}{|c|c|c|c|c|c|c|c|}
\hline & \multirow[t]{2}{*}{ Pre } & \multirow[t]{2}{*}{$1^{\text {st }}$ post } & \multirow[t]{2}{*}{$2^{\text {nd }}$ post } & \multirow[t]{2}{*}{ Kruskal-Wallis } & \multicolumn{3}{|c|}{ Mann-Whitney } \\
\hline & & & & & $\begin{array}{l}\text { Pre vs } \\
1^{\text {st }} \text { post }\end{array}$ & $\begin{array}{l}\text { Pre vs } \\
2^{\text {nd }} \text { post }\end{array}$ & $\begin{array}{c}\text { 1st vs } \\
2^{\text {nd }} \text { post }\end{array}$ \\
\hline G & $2.64 \pm 0.49(2-3)$ & $0.91 \pm 0.53(0-2)$ & $0.82 \pm 0.96(0-3)$ & 0.001 & 0.001 & 0.001 & 0.504 \\
\hline $\mathrm{R}$ & $1.50 \pm 0.74(0-3)$ & $0.77 \pm 0.53(0-2)$ & $0.18 \pm 0.39(0-1)$ & 0.001 & 0.001 & 0.001 & 0.001 \\
\hline B & $2.59 \pm 0.50(2-3)$ & $0.41 \pm 0.50(0-1)$ & $0.68 \pm 0.57(0-2)$ & 0.001 & 0.001 & 0.001 & 0.098 \\
\hline$A$ & $2.64 \pm 0.49(2-3)$ & $0.46 \pm 0.59(0-2)$ & $0.68 \pm 0.57(0-2)$ & 0.001 & 0.001 & 0.001 & 0.249 \\
\hline S & $0 \pm 0(0-0)$ & $0.45 \pm 0.21(0-1)$ & $0.45 \pm 0.21(0-1)$ & 0.602 & 0.317 & 0.317 & 0.946 \\
\hline
\end{tabular}

Pre: pre-operative period; $1^{\text {st }}$ post: post-operative period 1 week; $2^{\text {nd }}$ post: post-operative period 6 months.

ods were found $(\mathrm{p}=0.224)$. The VHI-10 score significantly improved in both the short- and long-term periods ( $\mathrm{p}=0.001$ at Kruskal-Wallis test). In the pre-operative period, the VHI-10 score was $17.77 \pm 3.76$; in the first post-operative period it was $7.09 \pm 5.27$, while in the second post-operative period it was $4.05 \pm 4.46$. Post-hoc analysis demonstrated significant differences between the pre- and the first post-operative periods $(\mathrm{p}=0.001)$, between the pre- and the second post-operative periods $(\mathrm{p}=0.001)$ and between the first and second post-operative periods $(\mathrm{p}=0.020)$. Only in one patient the VHI10 , which improved one week after phonosurgery passing from the pre-operative score of 15 to 9 , it return to 15 after 6 months.

All patients reported a slight to large improvement of voice quality and vocal fatigue one week after phonosurgery, while at 6 months after phonosurgery one patient reported no differences in vocal fatigue and voice quality compared to the pre-operative situation (as for the VHI-10). In particular, in the first post-operative period, a slight improvement in voice quality and vocal fatigue was reported in 7 of 22 patients, while substantial improvement was reported in the remaining 15 patients. These results were maintained after 6 months since in the second post-operative period a slight improvement in voice quality and vocal fatigue was reported in 6 of 22 patients, and large improvement was reported in 15 patients, while one patient reported no differences in vocal fatigue or voice quality compared to the pre-operative situation. However, no significant differences in perceived modification of voice between the first and second post-operative periods was found by Fisher's test $(p=0.919$ and $p=0.891$ for voice quality and vocal fatigue, respectively). In the patient who reported unchanged self-assessment of dysphonia 6 months following IL, we then performed a medialisation laryngoplasty (thyroplasty type 1) ${ }^{9}$ which resolved the problem of glottis insufficiency in the long-term.

\section{Discussion}

In the present study, the safety, feasibility and efficacy of trans-nasal fiberendoscopic IL with CAF in the treatment of GI due to UVFP was evaluated in a group of 22 patients. This is the first report on voice modification in a relatively large group of patients with UVFP treated with this phonosurgical technique, and assessed with a multidimensional set of measurements at 1 week and at 6 months after phonosurgery. The results appear promising and further support the applicability of this phonosurgical technique in the treatment of UVFP.

Specific findings are noteworthy. In particular, no complication during the surgical procedure or difficulties in the trans-nasal passage of the fiberscope were reported in any case. This could be related to several factors. First of all, autologous fat causes less inflammation in the injection site because it is less likely to cause an allergic reaction or local granuloma than synthetic materials ${ }^{81132}$. In addition, since the FEPS technique provides direct access to the surgical site and does not require external injection, oedematous complications are infrequent. As for the feasibility of IL under FEPS is concerned, the surgical procedure was performed in all patients, and each patient tolerated well both the liposuction and the phonosurgery. The intravenous injection of 1-2 mg midazolam decreases the anxiety of the patient, improving his/her collaboration, as in oesophageal-gastric and colon endoscopic procedures. The use of an $80 \mathrm{~cm}$ long flexible needle is possible because a 14 gauge Chiba needle is used for liposuction, instead of the Coleman cannula which draws very large blocks of fat tissue; moreover, the high-pressure pistol and 19 gauge flexible needle allow easy and safe passage of adipocytes.

As far as the efficacy of this phonosurgical technique in the treatment of GI due to UVFP is concerned, our results suggest that this IL provides good results not only in the short-term period, but also 6 months afterward. Previous 
studies using magnetic resonance ${ }^{33}$ showed that the injected fat that remains after 6 months may be considered permanent; therefore, the results at 6 months after IL may suggest that a positive voice outcome is maintained in the long-term. This could be explained by the fact that the stem cells contained in the autologous fat, in particular when it is centrifuged ${ }^{23-25}$, may develop into new adipocytes which replace the adipocytes that are deteriorated by the injection procedure. In this way, after the volume decrease due to partial reabsorption of the injected fat, the remaining fat is alive at the site of injection. In order to compensate for the partial reabsorption of the injected fat, twice the amount of material sufficient for achieving complete glottic closure is injected.

Regarding laryngostroboscopic findings, the results demonstrated significant improvement of all parameters (morphology of the vocal fold, position of the immobile vocal fold, periodicity of glottic vibration, glottic closure) both one week and 6 months after phonosurgery, with no significant changes between the first and the second post-operative periods. Also, for perceptual evaluation of dysphonia and in particular for the G, R, B, A parameters of the GRBAS scale (the $S$ parameter was obviously normal also before surgery) and for the maximum phonation time (MPT), the results showed significant improvement at both one week and 6 months after phonosurgery, with no significant changes between the first and the second post-operative periods.

As far as self-assessment of voice related QoL with the VHI-10 and of the perceived modification of voice after phonosurgery with the comparative self-assessment pre-post treatment, our results demonstrated a positive evolution of dysphonia after phonosurgery. Moreover, we found a significant difference between the VHI-10 obtained in the first and second post-operative periods; this seems to suggest progressive improvement of voicerelated QoL in the long-term and might be related to the regenerative potential of the stem cells included in the injected centrifuged autologous fat ${ }^{8112425}$. In the second period, 6 months after phonosurgery, one patient reported an unchanged situation of voice quality and voice fatigue compared to the pre-operative situation; the score of his VHI-10, which improved one week after phonosurgery, at 6 months returned to the same pre-operative score. In this patient, we then performed a medialisation laryngoplasty (thyroplasty type 1) ${ }^{9}$, which resolved the problem of GI in the long-term; this is a more invasive phonosurgical procedure, but has to be considered as a permanent technique.

The results reported herein appear similar to those of Saibene et al. ${ }^{11}$ who reported complete glottic closure in 2 of 3 patients with UVFP after trans-nasal IL using micro-fractured (non centrifuged) autologous fat; regarding perceptual evaluation of dysphonia with the GRBS scale, MPT and VHI-10, he reported an improvement in all 3 patients in his preliminary study. Trask et al. ${ }^{15}$ reported a reduction in medialisation of $20 \%$ to $40 \%$ as the injected aqueous fluid of the Cymetra was absorbed, in a group of 20 patients with UVFP treated with trans-nasal IL using Cymetra. Recently, Benninger et al al. ${ }^{34}$ reported good long-term results for VHI and MPT in 50\% of 25 patients with UVFP treated with trans-oral IL with non-centrifuged autologous fat under general anaesthesia.

Pagano et al. ${ }^{35}$ treated 18 patients with UVPF with transoral IL with non-centrifuged autologous fat under general anaesthesia, reporting significant improvement of glottic closure, MPT, VHI and GRBAS scores both in the shortand in the long-term after phonomicrosurgery. Umeno et al. ${ }^{36}$ treated 41 patients with UVFP with the same phonosurgical technique reporting significant improvement of MPT. Havas and Priestley ${ }^{37}$ followed 45 cases treated for UVFP with the same phonosurgical technique during a mean follow-up of 33 months, reporting a near normal voice in $87 \%$ of the patients. Fang et al. ${ }^{38}$ treated 33 patients with UVFP with the same phonosurgical technique, reporting an improvement of both voice and quality of life in $89 \%$ of patients after 12 months of follow-up. Cantarella et al. ${ }^{25}$ noted a significant improvement in glottic closure, MPT, VHI and GRBAS scores over a follow-up of 10.6 months in a group of 14 patients with GI due to UVFP or VF atrophy and treated with trans-oral IL under general anaesthesia with centrifuged autologous fat.

Our results appear similar to the results obtained by other authors using a trans-oral approach under general anaesthesia for IL with autologous fat in the treatment of UVFP. Unlike prior phonosurgical techniques for IL with autologous fat, this approach is carried out in the awake patient under LA, safely and with good efficacy, without the added risks of general anaesthesia or direct microlaryngoscopy. The use of autologous fat suggests performing IL in the OR in day-hospital (while IL with hyaluronic acid is office-based) because sterile manipulation of fat is required, although an anaesthesiologist is not required. By the fact that the patient is in the OR, the monitoring is recommended to control eventual vagal nerve reflexes during the procedure. This phonosurgical technique requires an additional surgeon (or a trained nurse), equipment and training in injection techniques. It has benefits especially in patients with anaesthesia risks or with anatomical problems of the neck and/or teeth.

There are several limitations to this study. First, the number of patients is relatively small. Second, the pre-therapy 
post-therapy study design does not allow direct comparison of IL under FEPS with others using different surgical techniques to improve voice in patients with GI due to UVFP. Third, follow-up was relatively short; thus, the long-term results of this phonosurgical technique should be investigated in a future study. Finally, this is a single institution study; thus, feasibility, safety and efficacy should be analysed in other voice centres as well.

\section{Conclusions}

In conclusion, trans-nasal fiberendoscopic IL with centrifuged autologous fat appears to be a safe, feasible and efficacious phonosurgical technique for the treatment of GI due to UVFP and seems to provide stable outcomes over time. The procedure is minimally invasive, without significant morbidity or complications, and is relatively easy to perform. However, the patient's collaboration is required, because the larynx is not immobile and control of swallowing is essential. This procedure does not require general anaesthesia and can also be performed in patients with abnormal neck anatomy or dental problems. The use of a flexible operative endoscope provides a direct approach to the glottic plane, allowing the surgeon continuous evaluation of glottic patency and voice quality modifications during the phonosurgical procedure. Therefore, trans-nasal fiberendoscopic injection laryngoplasty with centrifuged autologous fat can be considered as a possible option for the treatment of glottic insufficiency due to unilateral vocal fold paralysis.

\section{References}

1 Lee YS, Kim YE, Kwon M, et al. Short-term treatment results for unilateral vocal fold palsy induced by mediastinal lesions. J Voice 2014;28:809-15.

2 Cantarella G, Dejonckere P, Galli A, et al. A retrospective evaluation of the etiology of unilateral vocal fold paralysis over the last 25 years. Eur Arch Otorhinolaryngol 2017;274:347-53.

3 Benninger MS, Gillen JB, Altman JS. Changing etiology of vocal fold immobility. Laryngoscope 1998;108:1346-50.

4 Spataro EA, Grindler DJ, Paniello RC. Etiology and time to presentation of unilateral vocal fold paralysis. Otolaryngol Head Neck Surg 2014;151:286-93.

5 Baba M, Natsugoe S, Shimada M, et al. Does hoarseness of voice from recurrent nerve paralysis after esophagectomy for carcinoma influence patient quality of life? J Am Coll Surg 1999;188:231-6.

6 Barbu AM, Gniady JP, Vivero RJ, et al. Bedside injection medialization laryngoplasty in immediate postoperative patients. Otolaryngol Head Neck Surg 2015;153:1007-12.

7 Walton C, Conway E, Blackshaw H, et al. Unilateral vocal fold paralysis: a systematic review of speech-language pathology management. J Voice 2017;31:509.e7-.e22

8 Ricci Maccarini A, De Rossi G, Pieri F, et al. Injection laryngoplasty under fiberoptic endoscopy. In: Bergamini G, Presutti L, Molteni G, eds. Injection laryngoplastypp. Switzerland: Springer International Publishing; 2015. pp. 51-65.

9 Isshiki N, Morita H, Hiramoto M. Thyroplasty as a new phonosurgical technique. Acta Otolaryngol 1974;78:451-7.

10 Rosen CA, Amin MR, Sulica L, et al. Advances in officebased diagnosis and treatment in laryngology. Laryngoscope 2009;119:S185-212.

11 Saibene AM, Pipolo C, Lorusso R, et al. Transnasal endoscopic microfractured fat injection in glottic insufficiency. B-ENT 2015;11:229-34.

12 Anderson TD, Mirza N. Immediate percutaneous medicalization for acute vocal fold immobility with aspiration. Laryngoscope 2001;111:1318-21.

13 Damrose EJ. Percutaneous injection laryngoplasty management of acute vocal fold paralysis. Laryngoscope 2010;120:1582-90.

14 Friedman AD, Burns JA, Heaton JT, et al. Early versus late injection laryngoplasty in patients with potentially recoverable vocal fold paralysis. Laryngoscope 2010;120:2042-6.

15 Trask DK, Shellenberger DL, Hoffman HT. Transnasal, endoscopic vocal fold augmentation. Laryngoscope 2005;115:2262-5.

16 Andrade Filho PA, Carrau RL, Buckmire RA. Safety and cost-effectiveness of intra-office flexible videolaryngoscopy with transoral vocal fold injection in dysphagic patients. Am J Oto 2006;27:319-22.

17 Bove M, Jabbour N, Krishna P. Operating room versus office-based injection laryngoplasty: a comparative analysis of reimbursement. Laryngoscope 2007;117:226-30.

18 Diaz M, Riancho A, Borregan A. Chirurgia endolaringea fibroscopica (CELF). Rehabilitation vocal. Santander: Graficas Salima; 1999.

19 Ricci Maccarini A, Stacchini M, Salsi D, et al. Surgical rehabilitation of dysphagia after partial laryngectomy. Acta Otorhinolaryngol Ital 2007;27:294-8.

20 De Rossi G, Ricci Maccarini A, Borragan A, et al. Fonochirurgia fibroendoscopica. In: Schindler $\mathrm{O}$, ed. La voce. Padova: Piccin; 2009. pp. 433-42.

21 Koss SL, Baxter P, Panosian H, et al. Serial in-office laser treatment of vocal fold leukoplakia: disease control and voice outcomes. Laryngoscope 2017;127:1644-51.

22 Remacle M, Ricci Maccarini A, Matar N, et al. Reliability and efficacy of a new CO2 laser hollow fiber: a prospective study of 39 patients. Eur Arch Otorhinolaryngol 2012;269:917-21.

23 Coleman SR. Facial recountouring with lipostructure. Clin Plast Surg 1997;24:347-67.

24 Mazzola RF, Cantarella G, Torretta S, et al. Autologous fat injection to face and neck: from soft tissue augmentation to regenerative medicine. Acta Otorhinolaryngol Ital 2011;31:59-69. 
25 Cantarella G, Mazzola RF, Domenichini E, et al. Vocal fold augmentation by autologous fat injection with lipostructure procedure. Otolaryngol Head Neck Surg 2005;132:239-43.

26 Dejonckere PH, Bradley P, Clemente P, et al. A basic protocol for functional assessment of voice pathology, especially for investigating the efficacy of (phonosurgical) treatments and evaluating new assessment techniques. Guideline elaborated by the Committee on Phoniatrics of the European Laryngological Society (ELS). Eur Arch Otorhinolaryngol 2001;258:77-82.

27 Hirano M. Clinical examination of voice. New York: Springer-Verlag; 1981.

28 Ricci Maccarini A, Lucchini E. La valutazione soggettiva ed oggettiva della disfonia. Il protocollo SIFEL. Acta Phon Lat 2002;24:13-42.

29 Dejonckere P, Remacle M, Fresnel-Elbaz E, et al. Differentiated perceptual evaluation of pathological voice quality: reliability and correlations with acoustic measurements. Rev Laryngol Otol Rhinol 1996;3:219-24.

30 Forti S, Amico M, Zambarbieri A, et al. Validation of the Italian voice handicap index-10. J Voice 2014;28:263.e17-.e22.

31 Arffa RE, Krishna P, Gartner-Schmidt J, et al. Normative values for the voice handicap index-10. J Voice 2012;26:462-5.
32 Truzzi GM, Pauna HF, Bette P, et al. Methods of fat tissue processing for human vocal cord injection: a systematic review. J Voice 2017;31:244.e17-e21.

33 Brandenburg JH, Kirkham MW, Korschkee D. Vocal cord injection with autogenous fat: a long term magnetic resonance imaging evaluation. Laryngoscope 1996;102:495-500.

34 Benninger MS, Hanick AL, Nowacki AS. Augmentation autologous adipose injections in the Larynx. Ann Otol Rhinol Laryngol 2016;125:25-30.

35 Pagano R, Morsomme D, Camby S, et al. Long-term results of 18 fat injections in unilateral vocal fold paralysis. J Voice 2017;31:505.e1-e9.

36 Umeno H, Shirouzu H, Chutose S, et al. Analysis of voice function following autologous fat injection for vocal fold paralysis. Otolaryngol Head Neck Surg 2005;132:103-7.

37 Havas TE, Priestley KJ. Autologous fat injection laryngoplasty for unilateral vocal fold paralysis. ANZ J Surg 2003;73:938-43.

38 Fang T-J, Li HY, Gliklich RE, et al. Outcomes of fat injection laryngoplasty in unilateral vocal cord paralysis. Arch Otolaryngol Head Neck Surg 2010;136:457-62. 